Journal of The Electrochemical Society, 2007, Volume 154, Issue 6, Pages C312-C317.

Print ISSN: 0013-4651

Online ISSN: $1945-7111$

DOI: $10.1149 / 1.2722533$

http://scitation.aip.org/JES

http://scitation.aip.org/journals/doc/JESOAN-ft/vol_154/iss_6/C312_1.html

(C) The Electrochemical Society, Inc. 2007. All rights reserved. Except as provided under U.S. copyright law, this work may not be reproduced, resold, distributed, or modified without the express permission of The Electrochemical Society (ECS). The archival version of this work was published in Journal of The Electrochemical Society, 2007, Volume 154, Issue 6, Pages C312-C317.

\title{
Electrochemical Behavior of AISI 304SS with Particulate Silica Coating in 0.1 $\mathrm{M} \mathrm{NaCl}$
}

\section{Eiji Tada and G. S. Frankel}

Fontana Corrosion Center, Department of Materials Science and Engineering, The Ohio State University, Columbus, Ohio 43210, USA

This paper presents electrochemical behavior of AISI 304 stainless steel with a silica layer in a stagnant bulk solution of $0.1 \mathrm{M} \mathrm{NaCl}$. Layers composed of densely packed $350 \mathrm{~nm}$ diam silica particles were deposited cathodically on stainless steel at a constant voltage by electrophoretic deposition (EPD). Quite smooth and crackfree silica layers less than about $80 \mu \mathrm{m}$ in thickness were obtained and the thickness of the layer depended linearly on the deposition time. It is proposed that silica layers deposited by EPD can be used as simulated particulate layers to investigate localized corrosion of corrosion-resistant alloys under atmospheric environments. Electrochemical properties of silica-coated stainless steel samples in $0.1 \mathrm{M} \mathrm{NaCl}$ were investigated. The cathodic polarization behavior depended on the thickness of the silica layer; the limiting current density for oxygen reduction reaction decreased with increasing silica layer thickness. The effect of the silica layer on anodic polarization behavior was not remarkable.

One type of atmospheric corrosion involves the interaction of a humid environment with a contaminated surface. Water from the humid environment can be absorbed onto surfaces covered with fine dust particles containing soluble salts, creating a saturated deliquescence brine layer. ${ }^{1-4}$ Such a layer could be extremely corrosive, even to corrosion-resistant alloys (CRAs) if the temperature were high enough, due to the salt concentration and high availability of oxygen. Furthermore, it is possible that precipitates or rock particulates could act as a crevice former and cause localized corrosion of the substrate metal. The objective of this work is to generate, in a reproducible and controllable fashion, simulated artificial deliquescence brine layers and to investigate localized corrosion behavior of CRAs under such an environment.

In this study, electrophoretic deposition (EPD) of silica particles suspended in solution was utilized to create simulated particulate layers on the surface of CRAs. EPD is a very useful technique to deposit ceramic particles on metal surfaces; one can get ceramic layers with thicknesses on the order of $\mu \mathrm{m}$ to $\mathrm{mm}$ by EPD. ${ }^{5-7}$ Furthermore, the thickness of the ceramic layers can be controlled easily by changing the applied voltage or deposition time. Therefore, EPD can be utilized to create simulated particulate layers on CRAs in a reproducible and controllable fashion.

In general, when EPD of ceramic particles is conducted on a metallic substrate, such as stainless steel (SS), the substrate should be polarized either at a positive voltage or a negative voltage with respect to the counter electrode, depending on the zeta potential or isoelectric point of the particles to be deposited and the $\mathrm{pH}$ of the suspension. Because the isoelectric point for silica is quite acidic, 8 , silica particles in a neutral suspension carry negative charges on their 
surfaces. In this case, the substrate should be polarized to a positive voltage to deposit the silica particles, which might cause corrosion of the substrate. Even if EPD is conducted cathodically in an acidic suspension, the substrate also suffers from corrosion due to poor passivity in acidic solution. Hasegawa et al. performed excellent work to overcome these problems. ${ }^{10}$ A cationic polymer surfactant, polyethyenimine (PEI), was used to alter the surface charge on silica particles in a neutral suspension from negative to positive by adsorbing on the particle surface. As a result, it was possible to use cathodic EPD of silica on SS from a suspension of neutral $\mathrm{pH}$. In this study, cathodic EPD was carried out on SS according to this method to create silica layers in a reproducible and controllable fashion. The electrochemical behavior of SS electrodes coated with EPD silica layers was investigated in an aqueous solution containing chloride ions. In addition, the silica layers created by EPD were evaluated to assess if they can be utilized as host dust layers for the investigation of localized corrosion behavior of corrosion-resistant alloys under a thin water layer with dust particles. A subsequent publication will describe experiments on silica-coated SS electrodes under thin electrolyte layers.

\section{Experimental}

Preparation of silica-coated 304SS by EPD.- Spherical silica particles used for EPD in this study were prepared by the Stöber method, in which spherical silica particles are obtained by hydrolysis and condensation reactions of silicon alkoxides in an alcohol solvent in the presence of base and $\mathrm{H}_{2} \mathrm{O} .{ }^{11}$ In this study, spherical silica particles were synthesized by hydrolysis of tetraethoxysilane (TEOS, 99.5\%, Alfa Aeser) with a mixture of $\mathrm{H}_{2} \mathrm{O}$ and ethanol (EtOH), according to the procedure proposed by Nishimori et al. The mole ratio of TEOS/ $\mathrm{H}_{2} \mathrm{O}$ (added by $0.5 \mathrm{M} \mathrm{NH}_{4} \mathrm{OH}$ )/EtOH was 1:50:50. Sodium dodecyl sulfate (SDS, 99\%, Fisher Scientific) also was added to the mixture at a concentration of $0.02 \mathrm{wt} \%$ to act as a dispersant. Then, the mixture was stirred at room temperature for $24 \mathrm{~h}$, resulting in nucleation and growth of spherical silica particles. The color of the mixture changed from colorless to white during stirring, indicating that a silica suspension was produced. Silica particles were extracted from the suspension with a centrifuge (Allegra 64R, Beckman Coulter) and then dried at $120^{\circ} \mathrm{C}$.

The silica suspension for EPD on SS was a mixture of $\mathrm{H}_{2} \mathrm{O}$ and EtOH containing 2 wt \% silica particles prepared by the procedure described above. The mole ratio of $\mathrm{H}_{2} \mathrm{O}$ and $\mathrm{EtOH}$ was 1:10. Furthermore, $0.01 \mathrm{wt} \%$ polyethyleneimine (PEI, Aldrich) was added to the suspension to shift the isoelectric point of the silica particles, as described above., ${ }^{4,5}$ The suspension was agitated well in an ultrasonic bath for $1 \mathrm{~h}$ just before EPD. The samples used in this study were coupons of AISI $304 \mathrm{SS}(304 \mathrm{SS}, 1 \times 1 \mathrm{~cm})$. The samples were embedded in epoxy resin, polished with abrasive papers to 1200 grit, and ultrasonically cleaned with EtOH before EPD. EPD of silica particles was carried out cathodically (the sample worked as a cathode) at $29 \mathrm{~V}$ relative to a Pt sheet anode positioned above the sample. After EPD, the sample was removed from the EPD suspension and dried by air. Finally, the sample with a silica layer was stored in a desiccator before the electrochemical experiments.

Characterization of silica layer. - The silica layer deposited on 304SS by EPD was characterized by X-ray diffractometry (XDS-2000, Scintag) and scanning electron microscopy (SEM, Quanta, FEI). The thickness of the silica layers was measured using the difference in focal position on the substrate (304SS) and the top of the layer with optical microscope. 


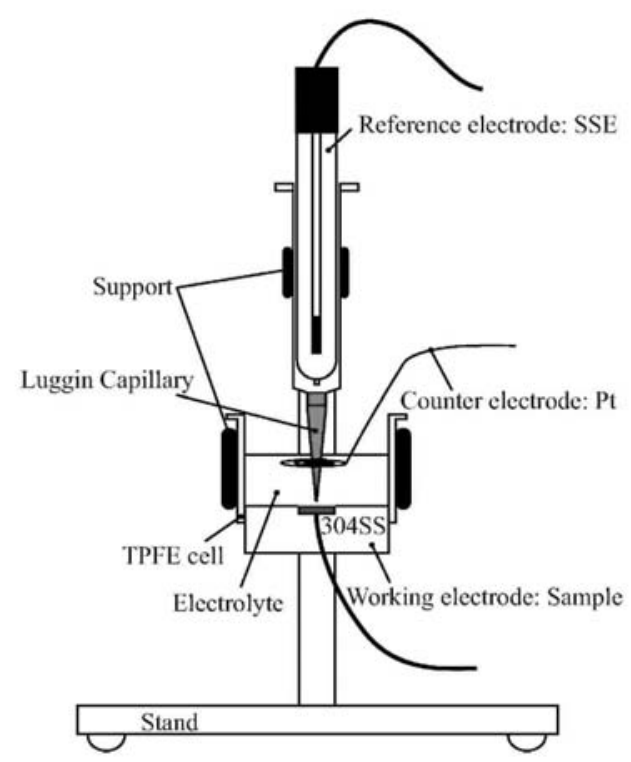

Figure 1. Experimental setup for electrochemical experiments with silica-coated 304SS.

Electrochemical measurement. - Figure 1 shows the experimental setup for electrochemical measurements of the silica-coated SS. A PTFE mold was used as a vessel. All the electrochemical experiments described in this study were carried out by a conventional threeelectrode method. The working electrode, preparing as described above, was a 304SS sample embedded in epoxy resin and coated with a silica layer. The counter electrode was a Pt wire spiral facing the sample surface. The reference electrode was a commercial $\mathrm{Ag} / \mathrm{AgCl}$ in $4 \mathrm{M} \mathrm{KCl}$ $\left[\mathrm{SSE}, \mathrm{E}_{\mathrm{SSE}}=0.197 \mathrm{~V}\right.$ vs standard hydrogen electrode (SHE)] connected to the electrochemical cell with a Luggin capillary. In this study, all potentials are referred to the SSE. The test solution was $0.1 \mathrm{M} \mathrm{NaCl}$. Electrochemical impedance spectra were measured at open-circuit potential using a potentiostat (263A, PAR) and a frequency response analyzer (1250, Solartron).

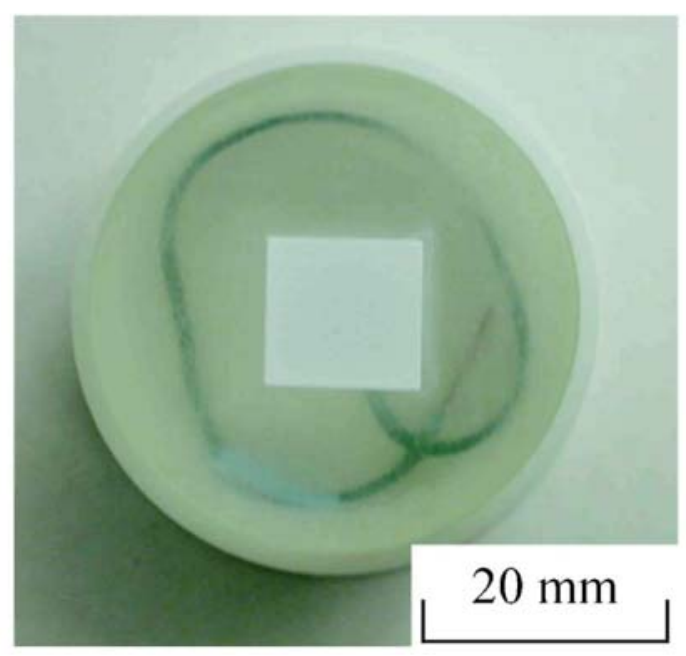

Figure 2. (Color online) An example of a sample of silica-coated 304SS. The deposition was conducted for $30 \mathrm{~s}$ at a constant potential of $29 \mathrm{~V}$. 


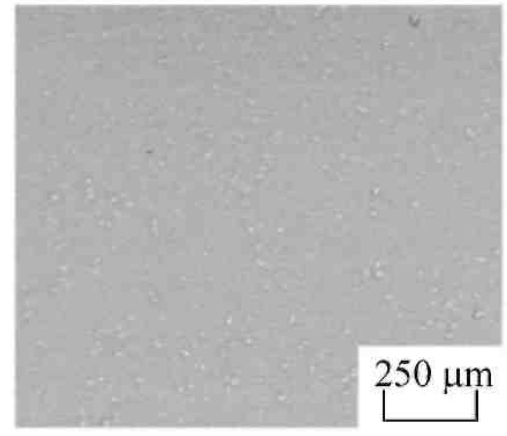

(a)

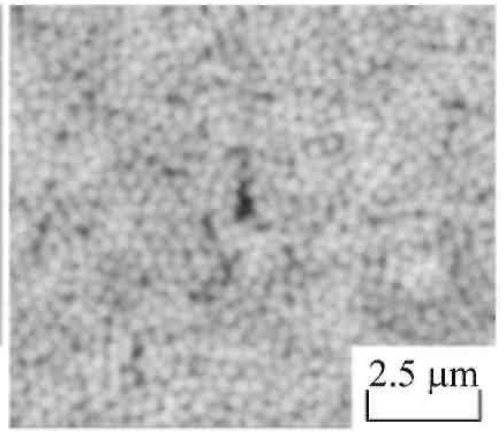

(b)

Figure 3. SEM micrographs of the surface of a silica-coated 304SS: (a) low magnification and (b) high magnification.

The ac potential amplitude was $10 \mathrm{mV}$, and the frequency was varied from $20 \mathrm{kHz}$ to $1 \mathrm{mHz}$. Potentiodynamic polarization curves were measured at 0.167 and $0.5 \mathrm{mV} / \mathrm{s}$ for anodic polarization and cathodic polarization, respectively. Cathodic current transients for silica-coated samples were measured at a potential of $-0.7 \mathrm{~V}$.

All the electrochemical experiments were conducted under open-to-air conditions at room temperature. All the solutions used in this study were prepared with analytical-grade chemicals and deionized water.

\section{Results}

Silica layer deposited on 304SS by EPD. - Figure 2 shows a photograph of an SS sample coated with a silica layer deposited for $30 \mathrm{~s}$. The white-colored coating was quite smooth and homogeneous. For further investigation of the surface of the silica layer, SEM observation was performed on a sample coated with a silica layer deposited for $5 \mathrm{~s}$. As shown in Fig. 3a, the silica layer had no defects such as cracks. Some large aggregated particles were randomly precipitated, which might be due to gravity sedimentation. At high magnification, as shown in Fig. 3b, the silica layer is seen to consist of very fine spherical particles packed densely. The particles obtained by the procedure in this study are monodispersed with a diameter of about $350 \mathrm{~nm}$. To characterize the structure of the silica layer deposited on 304SS, X-ray diffraction was performed with samples deposited for varying deposition times. In all the X-ray diffraction patterns in Fig. 4 , three sharp peaks around $2 \theta=43,45$, and $51^{\circ}$ are observed. These three peaks originated from the 304SS substrate. A broad peak around $2 \theta=22^{\circ}$ seen in all the patterns corresponds to amorphous silica. The integrated intensity of the broad peak increases with increasing deposition time, indicating that the thickness of the silica layer increased with the deposition time. This result is confirmed in Fig. 5, which shows the change in the silica layer thickness deposited on 304SS as a function of deposition time. Solid and open symbols denote the thickness of silica layers deposited from different batches of suspension. It can be seen clearly that the silica layer thickness is proportional to the deposition time. However, for deposition times longer than $120 \mathrm{~s}$, the deposited silica layer peeled off the substrate during evaporation of the suspension because of shrinkage of the silica layer. Therefore, in this study, the silica layers prepared by EPD used deposition times less than $120 \mathrm{~s}$, which is associated with a maximum silica layer thickness of about $80 \mu \mathrm{m}$, as seen in Fig. 5. 


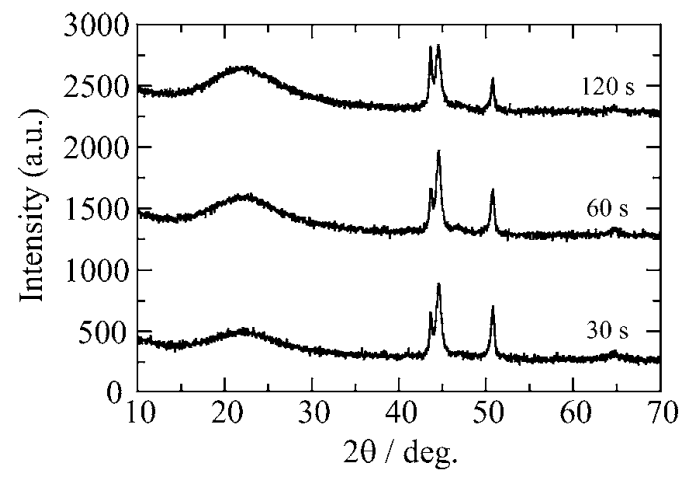

Figure 4. X-ray diffraction patterns of silica-coated 304SS with varying deposition time.

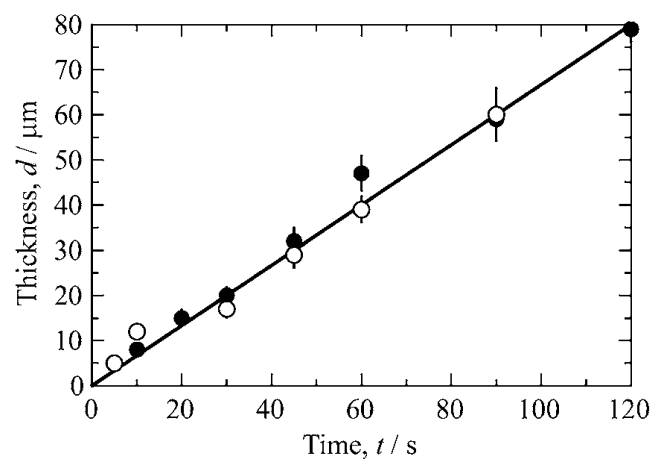

Figure 5. Thickness of silica layer on 304SS as a function of deposition time. The line represents least-squares fitting of the data.

Open-circuit potential and electrochemical impedance spectroscopy for silica-coated samples. - The open-circuit potential (OCP) for silica-coated 304SS was monitored for $1800 \mathrm{~s}$ after immersion in a stagnant bulk $0.1 \mathrm{M} \mathrm{NaCl}$ solution open to air. The OCP increased slowly, changing by about $100 \mathrm{mV}$ during the $1800 \mathrm{~s}$. As seen in Fig. 6, the OCP for silica-coated 304 SS was independent of the silica layer thickness in a mild condition like this experiment.

Electrochemical impedance spectroscopy (EIS) was also used to evaluate the silicacovered SS electrodes. The tests were performed at OCP (zero bias) in a stagnant bulk solution of $0.1 \mathrm{M} \mathrm{NaCl}$. Figure 7 shows Nyquist plots measured for uncoated and silica-coated samples with varying silica layer thickness. As seen in Fig. 7, the impedance tended to increase with increasing silica layer thickness, though the trend was not perfect.

Polarization behavior of silica-coated samples. - Anodic and cathodic polarization behavior of silica-coated 304SS was investigated. Figure 8 shows cathodic polarization curves for 304SS with varying silica thickness measured in a stagnant bulk $0.1 \mathrm{M} \mathrm{NaCl}$ solution. All of the cathodic polarization curves exhibited a limiting current region associated with the oxygen reduction reaction (ORR), followed by a current increase due to the hydrogen evolution reaction (HER). The ORR limiting current region for all of the silica-coated samples was in the potential range from -0.4 to $-0.8 \mathrm{~V}$, which is shifted in the noble direction by $100 \mathrm{mV}$ compared to the uncoated sample. The reasons for the higher ORR currents in the activation-controlled region and the higher HER currents are not known. 


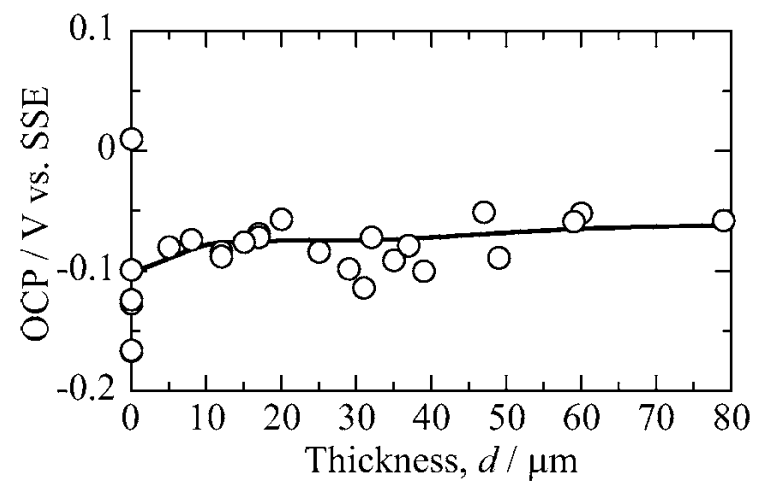

Figure 6. Relationship between OCP after $1800 \mathrm{~s}$ in $0.1 \mathrm{M} \mathrm{NaCl}$ solution and the silica layer thickness on 304SS.

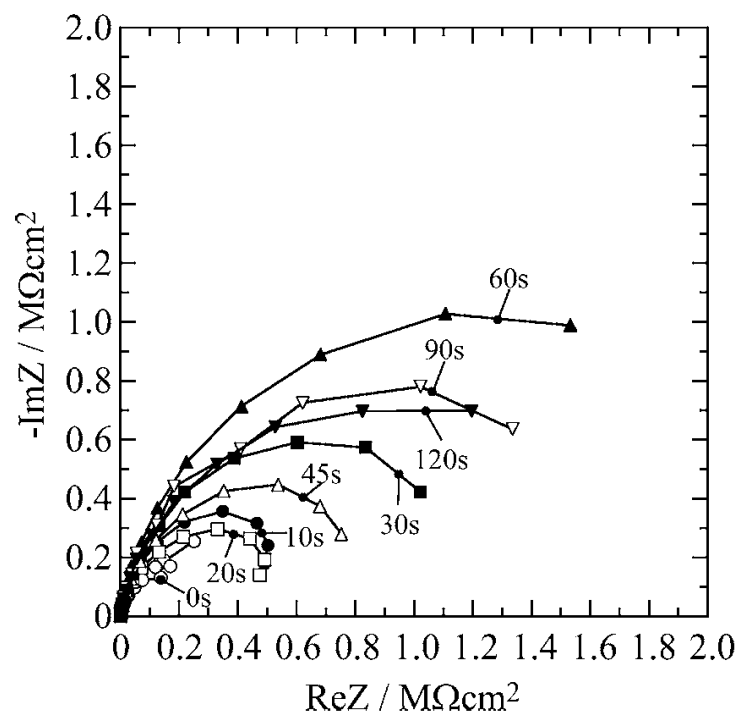

Figure 7. Nyquist plots of silica-coated 304SS with varying thickness measured at OCP in $0.1 \mathrm{M} \mathrm{NaCl}$. The time for each plot refers to silica deposition time, which is proportional to silica layer thickness.

To measure stable currents in the ORR limiting current region, current transients for uncoated and silica-coated samples were measured at a constant potential of $-0.7 \mathrm{~V}$ in a stagnant bulk $0.1 \mathrm{M} \mathrm{NaCl}$ solution. Figure 9 shows $\log -\log$ plots of the current transients. Within the first $30 \mathrm{~s}$ the currents decayed at a constant rate with a slope of about -0.4 , and the slope was almost independent of the silica layer thickness. A slope of -0.5 is predicted from Sand's equation. ${ }^{13}$ The time to achieve a stable current was longer and the stable current was smaller as the silica layer thickness increased. Figure 10 shows the relationship between the stable limiting current densities measured at $-0.7 \mathrm{~V}$ and the reciprocal of the silica layer thickness. It is apparent that the limiting current increased approximately linearly with the reciprocal of the silica layer thickness for layers thicker than about $12.5 \mu \mathrm{m}$; the limiting current did not change very much for silica layer thickness less than $12.5 \mu \mathrm{m}$. The $12.5 \mu \mathrm{m}$ thickness roughly corresponds to several tens of silica particles aggregated as a layer. These results demonstrate that when the silica layer thickness is less than about $12.5 \mu \mathrm{m}$, the barrier effect of the silica layer on diffusion of dissolved oxygen should be negligible. 


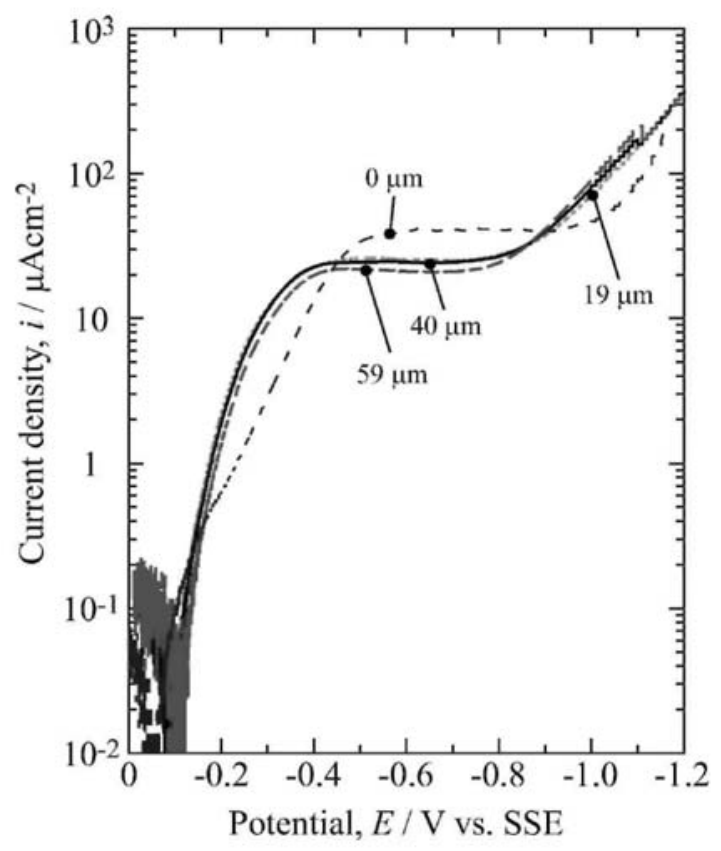

Figure 8. Cathodic polarization curves for silica-coated 304SS with different silica layer thickness.

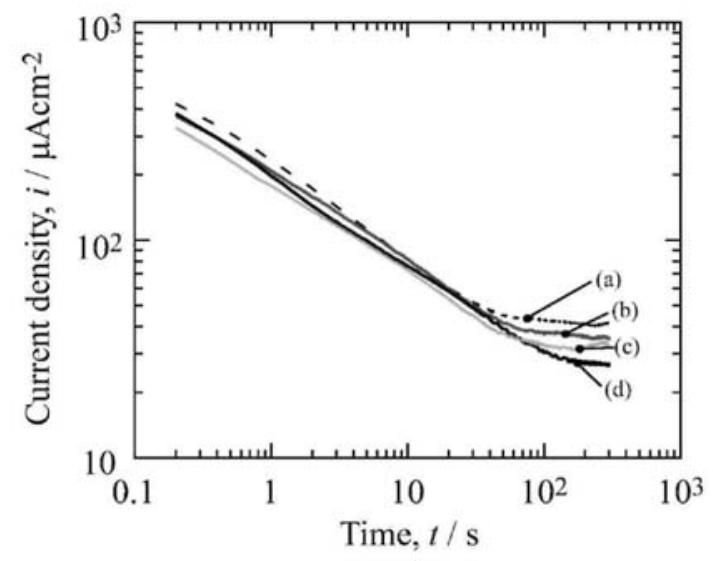

Figure 9. Log-log plots of current decays for 304SS with varying silica layer thickness at a constant potential of -0.7 V: (a) 0, (b) 20, (c) 39, and (d) $79 \mu \mathrm{m}$.

Anodic polarization behavior for 304SS coated with a silica layer was also investigated in a stagnant bulk solution of $0.1 \mathrm{M} \mathrm{NaCl}$ open to air. Figure 11 shows anodic polarization curves for uncoated and silica-coated samples with varying silica layer thickness. All the anodic polarization curves exhibited spontaneous passivity, followed by the emergence of metastable pitting spikes and then continuous growth of pitting corrosion. To characterize the anodic polarization curves, the passive current density at $0.25 \mathrm{~V}$ and breakdown potentials when the current density exceeded $50 \mu \mathrm{A}$ were extracted from the curves (Fig. 12). There seems to be no strong dependency of either parameter on silica layer thickness. These results suggest that passivity of 304SS in a stagnant bulk solution of $0.1 \mathrm{M} \mathrm{NaCl}$ is not changed in the presence of silica layers. 


\section{Discussion}

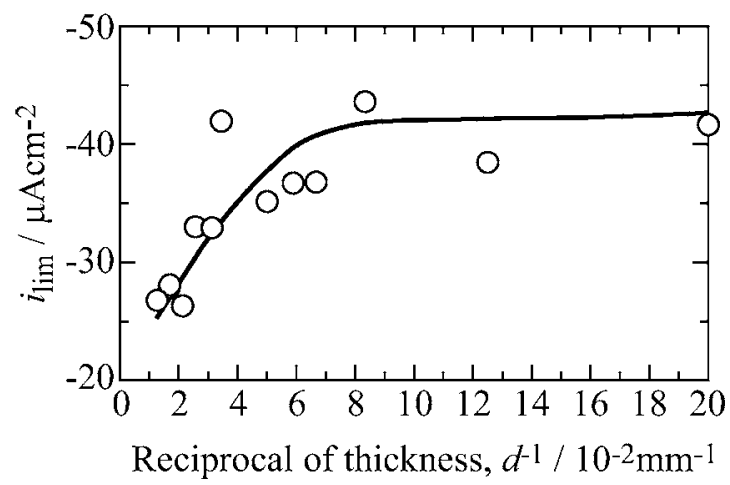

Figure 10. Limiting current density for silica-coated 304SS as a function of the reciprocal of silica layer thickness.

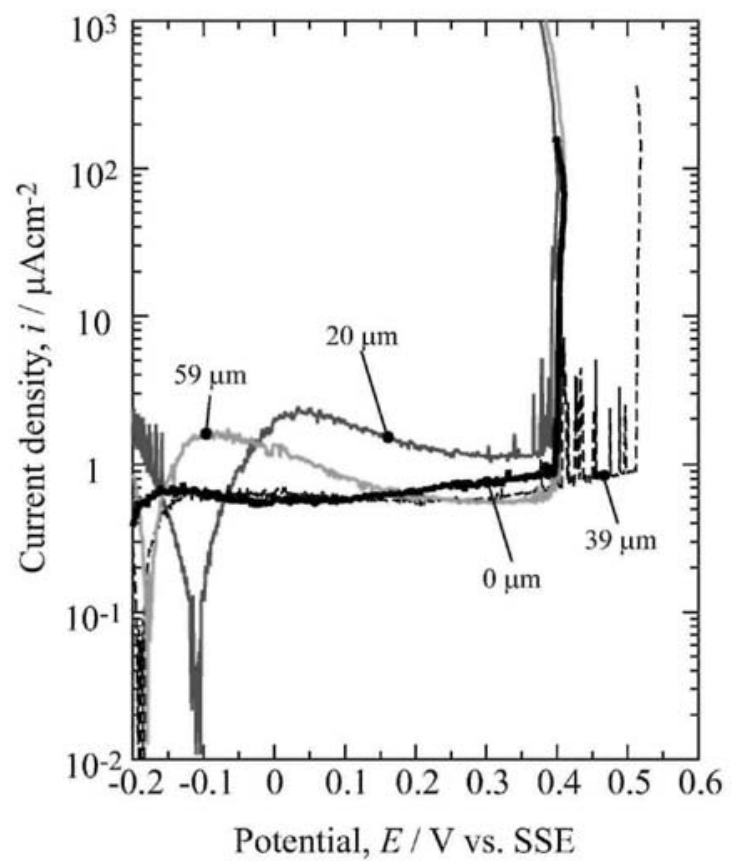

Figure 11. Anodic polarization curves for silica-coated 304SS as a function of the silica layer thickness.

Electrochemical interface on 304SS under a silica layer. - In this study it was observed that a smooth and homogeneous layer consisting of spherical silica particles about $350 \mathrm{~nm}$ in diameter can be deposited cathodically on 304SS using EPD and the thickness of the silica layer can be controlled with deposition time. As shown in Fig. 4, although the layer was composed of silica particles packed densely, there was spacing between the particles in the layer. Therefore, it can be expected that an electrolyte will penetrate through the spacing towards the surface of 304SS due to capillary condensation, resulting in the formation of electrochemical interface on the surface of 304SS. In addition, because silica has a hydrophilic nature, the silica layer will promote absorption of electrolytes. From these considerations, the electrochemical interface for 
silica-coated 304SS formed in a stagnant bulk solution of $0.1 \mathrm{M} \mathrm{NaCl}$ is schematically shown in Fig. 13. In this figure the silica layer is depicted with a uniform packing structure throughout the thickness from the surface of 304SS to the top of the silica layer. It could be argued that the packing structure of the layer deposited on 304SS should become less dense with increasing distance from the surface of 304SS because the driving force for deposition of silica particles should decrease with increasing silica layer thickness. However, because the silica deposition rate was almost constant, as shown in Fig. 5, it is considered that the packing structure did not change with silica layer thickness up to $80 \mu \mathrm{m}$.
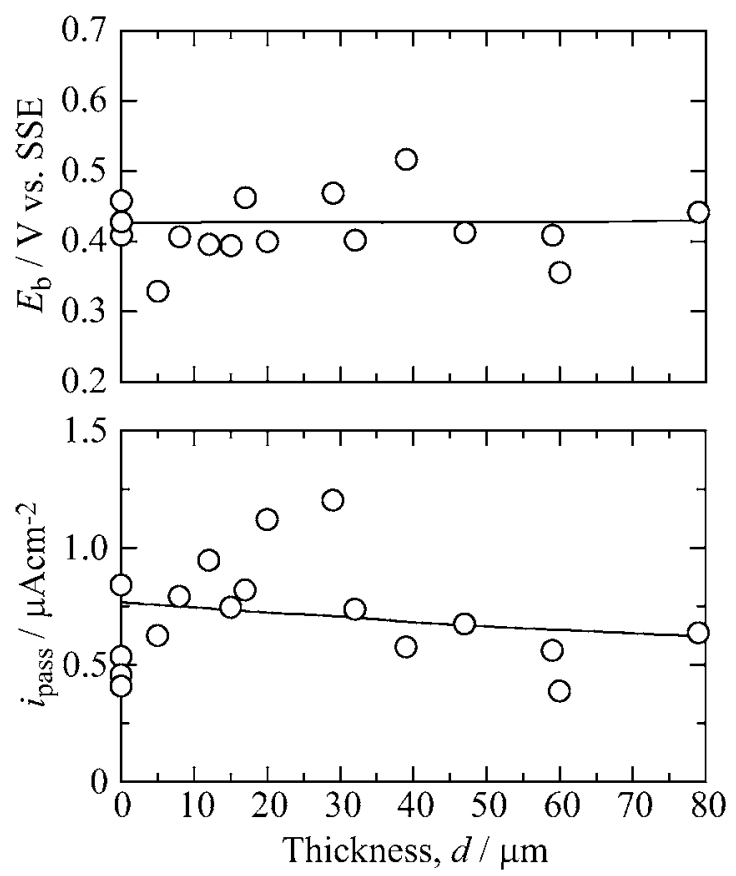

Figure 12. Breakdown potential $\mathrm{E}_{\mathrm{b}}$ and passive current density $i_{\text {pass }}$ of silica-coated 304SS as a function of the silica layer thickness. The lines represent trends in the data.

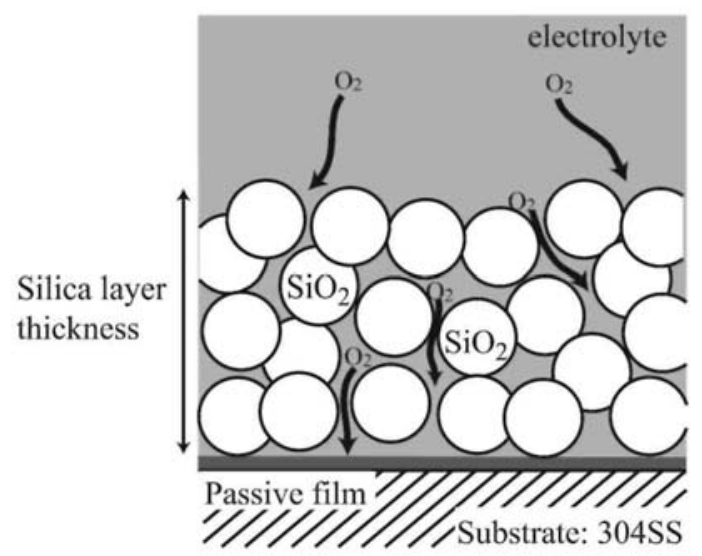

Figure 13. Schematic drawing of electrochemical interface for silica-coated 304SS in a stagnant bulk solution of 0.1 $\mathrm{M} \mathrm{NaCl}$. 
Effect of silica layer on electrochemical behavior of 304SS. - The silica layer deposited on 304SS was shown to inhibit the ORR, indicating that the silica layer acted as a barrier for diffusion of dissolved oxygen. As shown in Fig. 3b and schematically in Fig. 13, open space exists between neighboring silica particles, and it is considered that this interstitial space is filled with electrolyte when a silica-coated 304SS sample is immersed in solution. Any dissolved oxygen molecules would have to diffuse through the interstitial region to be reduced on the surface of 304SS. The diffusion path through the silica layer would be added to the thickness of the hydrodynamic boundary layer in the solution. As mentioned above, because the packing structure of the silica layer prepared in this study is considered to be uniform, the diffusion length for dissolved oxygen should increase linearly with silica layer thickness. The limiting current and the time to achieve the stable limiting current were in fact shown to depend on the silica layer thickness, as shown in Fig. 9 and 10. However, the barrier nature is insignificant when the silica layer thickness is less than about $12.5 \mu \mathrm{m}$, corresponding to a layer with $20-30$ particles.

The silica layers did not affect the passive dissolution rate of 304SS. Silica particles are coagulated on the working electrode by the imposed electric field during EPD. Therefore, it is considered that the silica particles are not chemically bonded to the passive surface. This likely is similar to the situation for a dust-covered surface. Because the chemical stability of the passive film should be independent of the presence of a silica layer, it is expected that the passive current density would not depend strongly on the silica layer thickness. The silica layer thickness also was observed to have little effect on the breakdown potential.

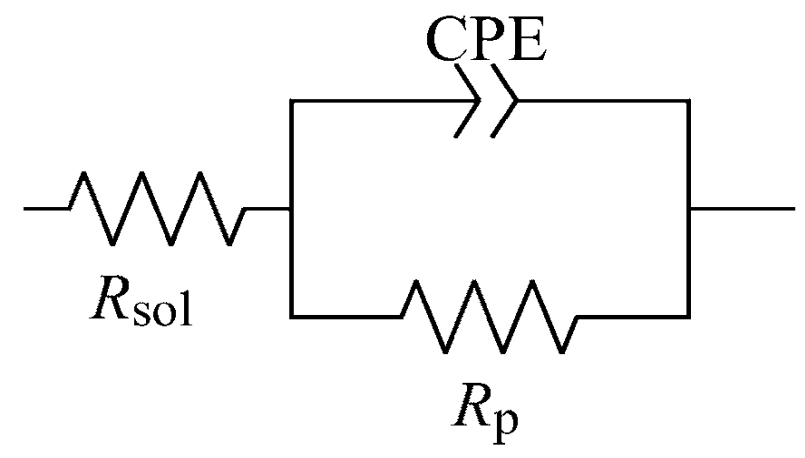

Figure 14. Equivalent circuit model for analyzing impedance data from 304SS with a silica coating.

As shown in Fig. 6, the OCP for silica-coated 304SS was about $-0.075 \mathrm{~V}$ independent of the silica layer thickness. Figure 8 shows that the cathodic reaction, which is considered to be the ORR, should not be in the limiting condition at the OCP. However, as shown in Fig. 11, the anodic reaction at OCP is passive dissolution, and the passive current density is quite low compared to the ORR limiting current density. In other words, the rate-determining step for corrosion of the silica-coated 304SS at OCP should be the anodic reaction. As mentioned above, the OCP should be almost independent of the silica layer thickness because the stability of the passive film seems to be independent of the presence of the silica layer. The electrochemical impedance for silica-coated 304SS at OCP generally increased with increasing silica layer thickness. This is because the electrochemical impedance for the cathodic reaction can be observed at OCP; the electrochemical impedance for the anodic reaction occurring in parallel 
with the cathodic reaction should be quite high, relating to a low passive current density. Therefore, it is concluded that EIS behavior shown in Fig. 7 is directly related to the barrier action of the silica layer on 304SS. By fitting impedance data of Fig. 7 with an equivalent circuit in Fig. 14, EIS parameters such as $R_{\text {sol }}, R_{p}$, and constant phase element (CPE) parameters can be obtained. Furthermore, from the CPE parameters obtained, an equivalent capacitance, $C_{\mathrm{eq}}$, is calculated with Eq. 1 by Hsu et al. as follows ${ }^{14}$

$$
C_{\mathrm{eq}}=A\left(2 \pi f_{m}\right)^{n-1}
$$

where $A, n$, and $f_{m}$ are CPE magnitude, CPE exponent, and the frequency value where $-\operatorname{Im}(Z)$ is maximum, respectively.

Figure 15a shows changes in $R_{\text {sol }}$ and $C_{\text {eq }}$ as a function of deposition time. It seems that $R_{\mathrm{sol}}$ is independent of deposition time over a range of $120 \mathrm{~s}$. It is considered that $R_{\text {sol }}$ is composed of two series resistances; one is a resistance between the capillary tip and the top surface of a silica layer, and the other is a resistance inside the silica layer. Because the silica layer is much thinner than the distance between the capillary tip and the top surface of the silica layer, the total solution resistance might not change much even when the resistivity inside the silica layer is larger than that outside the silica layer. The capacitance also varies little with increasing silica layer thickness, though the capacitance for the uncoated sample is the largest measured. The clearest trend in the impedance data is the increase in $R_{p}$ with silica layer thickness for layer thickness less than about $40 \mu \mathrm{m}$ (Fig. 15b). The lowest $R_{p}$ measured is for the uncoated sample. Even though the trends in $R_{p}$ and $C_{\text {eq }}$ are not the same, the trends suggest that the silica coating blocks or covers the surface where the electrochemical reactions take place to a small extent.

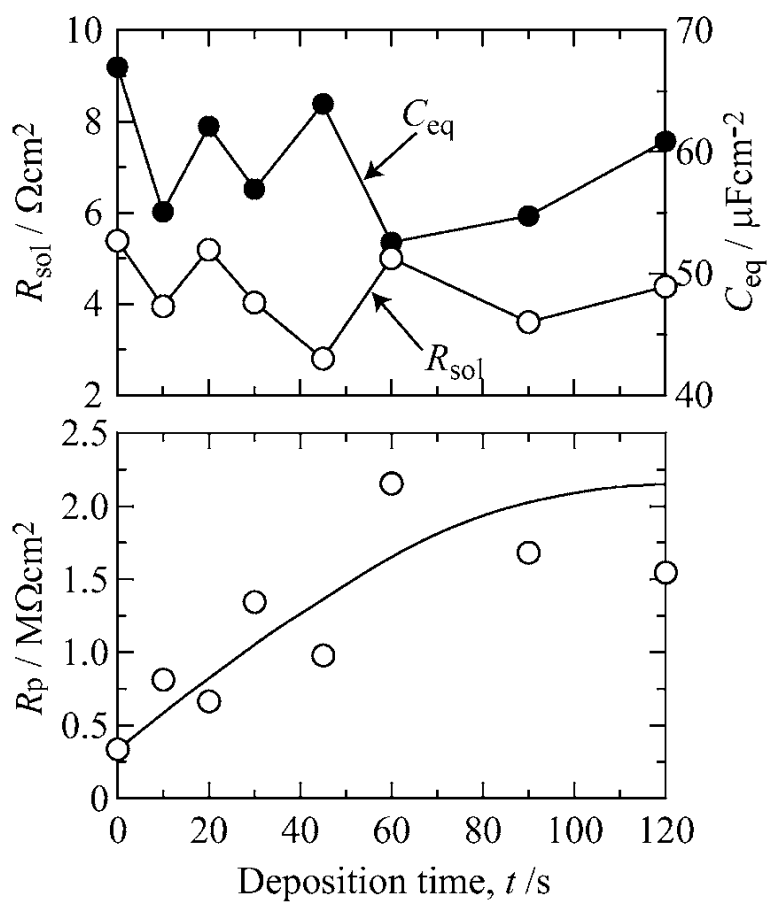

Figure 15. Effect of EPD deposition time on $C_{\mathrm{eq}}, R_{\mathrm{sol}}$, and $R_{p}$ in $0.1 \mathrm{M} \mathrm{NaCl}$. The line in the lower graph represents the trend in the data. 
Effect of silica layer on localized corrosion. - The breakdown potential for silica-coated 304SS was found to be independent of the presence of a silica layer. However, if localized corrosion is initiated on a silica-coated 304SS in a neutral electrolyte, it is possible that the silica layer might affect localized corrosion growth. The rate-determining step should be changed from anodic passive film dissolution to cathodic reduction of dissolved oxygen. In this case, the barrier effect of a silica layer for reduction reaction of dissolved oxygen should become important, and the silica layer could inhibit growth by reducing the ORR rate. However, the silica layer could act as a robust pit cover that would retard outward diffusion of dissolved cations and enhance acidification of the pit electrolyte. This phenomenon is essentially the stabilization of the localized attack because of the promotion of a crevicelike structure. The silica should be quite stable to the acidified pit electrolyte. There was no evidence found in this study to support the stabilization of crevice corrosion by the silica layer, but the role of the silica layer might become more important under atmospheric corrosion environments.

\section{Conclusions}

In this study, EPD was used to create particulate silica layers on 304SS that might simulate a rock particulate layer. The electrochemical behavior for silica-coated 304SS was investigated in detail in a stagnant bulk solution of $0.1 \mathrm{M} \mathrm{NaCl}$. The results obtained in this study are summarized as follows.

1. A homogeneous and smooth coating of silica particles was obtained cathodically on 304SS and the thickness of the silica layer was controlled up to $80 \mu \mathrm{m}$ by changing the deposition time. 2. The silica layer can be utilized in electrochemical tests as a simulated precipitate layer on a corrosion resistant alloy, such as SSs.

3. The silica layer inhibited the reduction reaction of dissolved oxygen because of an increase in diffusion length.

4. The silica layer had little effect of the passive current density and breakdown potential due to the stability of 304SS passive films.

\section{Acknowledgments}

The authors are grateful to Dr. R. Buchheit for fruitful discussions. Support by the Science and Technology Program of the Office of the Chief Scientist (OCS), Office of Civilian Radioactive Waste Management (OCRWM), U.S. Department of Energy (DOE), is gratefully acknowledged. The work was performed under the Corrosion and Materials Performance Cooperative, DOE Cooperative Agreement Number: DE-FC28-04RW12252. The views, opinions, findings, and conclusions or recommendations of authors expressed herein do not necessarily state or reflect those of the DOE/OCRWM/ OCS.

The Ohio State University assisted in meeting the publication costs of this article.

\section{References}

1. C. Leygraf and T. E. Graedel, Atmospheric Corrosion, p. 9, John Wiley and Sons, New York (2000).

2. R. E. Lobnig, D. J. Siconolfi, L. Psota-Kelty, G. Grundmeier, R. P. Frankenthal, M. Stratmann, and J. D. Sinclair, J. Electrochem. Soc, 143, 1539 (1996).

3. R. Lobnig, J. D. Sinclair, M. Unger, and M. Stratmann, J. Electrochem. Soc, 150, A835 (2003). 
4. M. Apted, F. King, D. Langmuir, R. Arthur, and J. Kessler, J. Optim. Theory Appl, 18, 43 (2005).

5. A. R. Boccaccini, J. A. Roether, B. J. C. Thomas, M. S. P. Shaffer, E. Chavez, E. Stoll, and E. J. Minay, J. Ceram. Soc. Jpn., 114, 1 (2006).

6. I. Zhitomirsky, Adv. Colloid Interface Sci., 97, 279 (2002).

7. O. O. Van der Biest, Annu. Rev. Mater. Sci., 29, 327 (1999).

8. H. Nishimori, M. Tatsumisago, and T. Minami, J. Ceram. Soc. Jpn., 103, 78 (1995).

9. H. Nishimori, M. Tatsumisago, and T. Minami, J. Sol-Gel Sci. Technol, 9, 25 (1997).

10. K. Hasegawa, M. Tatsumisago, and T. Minami, J. Ceram. Soc. Jpn., 105, 569 (1997).

11. W. Stöber, A. Fink, and E. Bohn, J. Colloid Interface Sci., 26, 62 (1968).

12. H. Nishimori, M. Tatsumisago, and T. Minami, J. Ceram. Soc. Jpn., 103, 743 (1995).

13. G. S. Frankel, L. Stockert, F Hunkeler, and H. Boehni, Corros. Sci., 43, 429 (1987).

14. C. H. Hsu and F Mansfeld, Corros. Sci., 57, 747 (2001). 